\title{
Modeling a Jitter in Telecommunication Data Networks for Studying Adequacy of Traffic Patterns
}

\author{
Aleksandr Vladimirovich Blagov ${ }^{1}$ \\ ${ }^{1}$ Samara State Aerospace University (SSAU), 34, Moskovskoye Shosse, Samara, 443086, Russia \\ Correspondence: Aleksandr Vladimirovich Blagov, Samara State Aerospace University (SSAU), Moskovskoye \\ Shosse, Samara 443086, Russia. E-mail: alexander.blagov@gmail.com
}

Received: October 30, 2014

Accepted: November 6, $2014 \quad$ Online Published: March 15, 2015

doi:10.5539/mas.v9n4p254

URL: http://dx.doi.org/10.5539/mas.v9n4p254

\begin{abstract}
This article focuses on researching quality of service in telecommunication networks, using simulation models. Due to the presence of the self-similarity effect in network traffic, the author used the approach with self-similar traffic patterns.

The article proposes the method associated with building a one-dimensional distribution of delay variation, a jitter. Design and analysis of delay variation are tested on modifications of telecommunications traffic models «Input $\mathrm{M} / \mathrm{G} / \infty$ » and «On-Off Sources», which make it possible to simulate traffic with pre-defined self-similar properties and at the same time with pre-defined statistical characteristics of small levels.
\end{abstract}

Software suite AnyLogic is used as simulation medium that supports approaches with both discrete and continuous time of simulation.

The experiments established the benefits of improved versions of «InputM/G/ $/ \infty$ » and «On-Off Sources» models over the base models in terms of the most important characteristic of Quality of Service in the jitter network. A conclusion was made that simulation modeling of the studied model makes it possible to analyze quality of service in a telecommunications network by such a parameter as delay variation.

Keywords: internet, telecommunication networks, traffic simulation, jitter, network delay, self-similar traffic, QoS

\section{Introduction}

The problem of predicting the behavior of traffic in telecommunication networks is particularly important due to the tremendous development of information networks.

With the advent of broadband networks, which are characterized by a mixture of different types of traffic (video, ftp, http ...), conventional methods for describing traffic become questioned. This type of traffic has strongly pulsating nature, which cannot be described by traditional models, such as the Poisson model. This phenomenon has a strong influence on networks design. New models that are characterized by this to the «explosion» effect, need analysis, design, planning and broadband networks overload management (Ramakrishnan, 1997, p. 29).

\subsection{Study of Traffic Models}

Traffic study on broadband networks confirmed the presence of the above phenomenon. Traffic with such features is called «self-similar» or «fractal» (Erramilli et al., 1996). Traffic in such a network has the so-called property of «self-similarity», i.e., it looks qualitatively uniform almost for any scale of the time axis, has memory (aftereffect) and is characterized by high level of Ts-Tpi (Park et al., 2000). It turned out that in the conditions of self-similar traffic, methods of calculating parameters of modern computer networks (channel capacity, buffer capacity, etc.) based on Poisson models and Erlang's formulas, which are successfully used for designing telephone networks, provide unduly optimistic solutions and lead to underestimation of the real needs of network resources (Simpson, 2004).

Thus, the problem of teletraffic self-similarity arose, which has been widely discussed in many works and that still has not lost its relevance.

Practically relevant is the problem of traffic analysis in a telecommunications network for building a simulation model of network traffic, taking into account the self-similarity and conventional statistical characteristics for its 
further use in programs of simulation modeling in studying real networks at the design stage with regard of Quality of Service. A good model can be used not only for traffic simulation, but also for identifying its structure, parameters of sources generating traffic, situation in the network over which the traffic is distributed, delay variations of a stream transmitted through the network, etc. However, there is still no single universally accepted model of self-similar traffic. Some known models do not feature the desired degree of self-similarity (Matrawy et al., 2002); others feature very high computational complexity (Melamed et al., 2003). From the statistical point of view, the distinction between traditional models of traffic and measured network traffic is interesting and important. There is evidence that the fractal properties of the traffic observed during the experiment (in particular, long-range dependence and «heavy tail» distribution) have a real impact on a wide range of problems arising in designing and development of networks (Shelukhin et al., 2003).

Currently, researchers offer many various models of self-similar network traffic (Park et al., 2000; Privalov \& Blagov, 2011; Mondragon et al., 2001). As a rule, for verifying adequacy and efficiency of a particular model, various statistical characteristics are offered, such as the Hurst parameter (describing the degree of self-similarity) (Gromov et al., 2007), mathematical expectation, the autocorrelation function and one-dimensional traffic distribution. Less common are estimates of average and maximum queue length (Mondragon et al., 2001). However, in many cases, for example in studying quality of service in the network, of great importance is evaluation of its most important characteristic, the delay variation, i.e., the jitter (Blagov, 2011).

\subsection{Studying Quality of Service}

Note that models that approximate the network traffic by several parameters simultaneously, e.g., by statistical characteristics of various levels, become increasingly important in practice due to the advent of quality of service requirements in today's networks (Shelukhin et al., 2003).

Quality of service - this term is used in the field of telecommunication networks for denoting the ability of a network to provide traffic parameters corresponding to those specified in traffic agreement (Vegesna, 2002).

In most cases, quality of connection is determined by four parameters:

- Bandwidth that describes the rated capacity of communication media determines channel width. Measured in bps, kbps, Mbps, Gbps.

- Packet Delay is measured in milliseconds.

- Oscillations (variation) of delay in packets transmission - jitter.

- Packet loss. Defines the number of packets lost in the network during transmission.

In particular, such QoS characteristic as jitter of a flow through a network node is determined by queue distribution at the particular node, where this stream competes for transmission to the other traffic in the same direction.

\section{Method}

\subsection{Jitter in Data Transmission Networks}

In addition to statistical characteristics of small levels in simulated traffic and natural traffic, from a practical point of view it is important to study quality of service in information networks using simulation models. The most important characteristic of QoS in this case is delay variation - jitter.

Jitter is inequality of periods of time allocated for package delivery. In other words, this is the variation of the maximum and minimum time of transferring a packet from the average (Dann, 2006).

Jitter or the so-called packet delay variation is an indicator of the quality of support of a number of services by the network (Privalov, 2000). Violation of jitter restrictions can lead to loss of quality of data transmission in telecommunication networks.

As a rule, jitter is determined using the following approach. Some source with constant rate transmits a deterministic (periodic) flow of packets into the network; in this flow packets follow each other at regular intervals $T$. We will call packets from this source «labeled». On their way across the network, labeled packets pass one or more network nodes that are statistical multiplexers (Figure 1). In the multiplexer, labeled packets compete for transmission with packets from other sources, which we will call «background packets», and the traffic up of such packages will be called «background traffic» (Privalov, 2007). After passing the last multiplexer on their way, labeled packets may arrive at their destination with varying delay, due to jitter (Figure 
2). Intervals between their arrivals can be defined as $L_{1}, L_{2}, \ldots, L_{k}$, i.e. $L_{i}$, where $i \in[1, k]$. Accordingly, the jitter of the i-th package in this case is the time difference of the labeled packet passage with average time $\left\{L_{i}-T\right\}$.

For modeling and studying the jitter in level to verify the adequacy of models of network traffic, for example for different versions of «Input $\mathrm{M} / \mathrm{G} / \infty$ » and «On-Off Sources» models described below, let us present source traffic in one case, and simulated traffic in the other case, as background flow. In both cases, let us introduce a constant rate source (Figure 1), flow of packets from which will be the «labeled» flow.

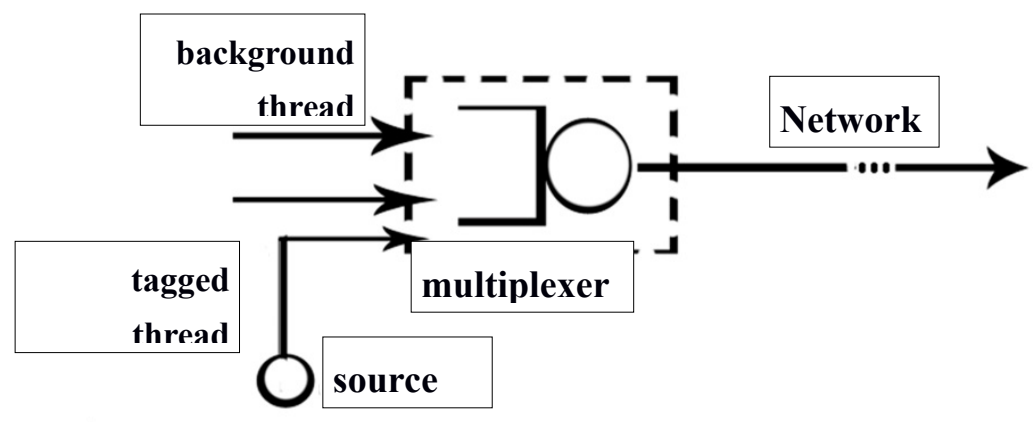

Figure 1. System with background and labeled flows

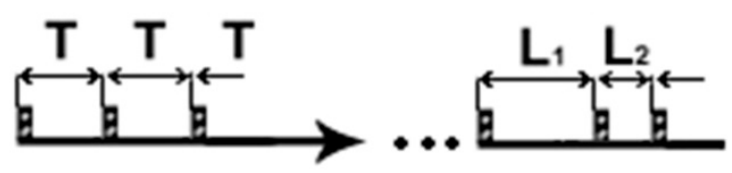

Figure 2. Changing time period between packets of labeled flow

\subsection{Modeling Network Traffic}

Model of network traffic «Input $\mathrm{M} / \mathrm{G} / \infty$ » is a model that is able to generate self-similar traffic (Privalov and Blagov, 2011). It is characterized by constant availability of traffic sources occurring in each time slot, which work for a certain period determined at the moment of source occurrence. Each source produces a number of information packets. In each time slot, traffic is defined as the total volume of all packets present in the system. Traffic sources are considered independent. Of course, in reality, especially in local area networks, this assumption is not always true, but it is made in level to describe a system comfortable enough for building computational algorithms for defining parameters of a system that generates network traffic.

In whole, the model «Input $\mathrm{M} / \mathrm{G} / \infty \gg$ is a good alternative to other existing network traffic models. It successfully simulates some widespread applications such as Telnet and FTP (Privalov and Blagov, 2011), and also is good for mathematical treatment.

Model «Input $\mathrm{M} / \mathrm{G} / \infty$ » is organized as follows: Let us assume that in a system with discrete time $t \in I_{-\infty}$ information is transmitted in packets of the same size, and the traffic $Y=\left(\ldots, Y_{-1}, Y_{0}, Y_{1}, \ldots\right)$ is a random process of discrete time, where $Y_{t}$ is the number of packets arriving at a certain point in a time step. These packages are

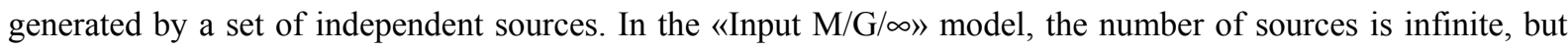
each of them generates packages during one final time interval, and then pauses.

In the model, new sources occur in the system in each time slot, forming a Poisson flow with parameter $\lambda$ :

$$
\operatorname{Pr}\left\{A_{t}=k\right\}=e^{-\lambda} \frac{\lambda^{k}}{k !}, \quad k \in Z_{+},
$$


where $A_{t}$ is the number of new traffic sources that have appeared in the system in time slot $t$. Each source has a finite time in the system, after which it disappears from the system. Working time of sources is a random value with a «heavy tail» distribution:

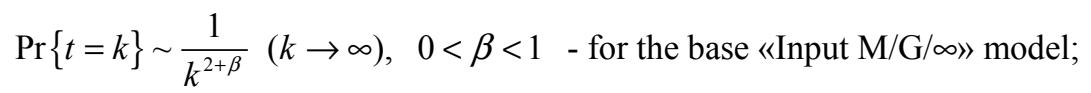

$$
\begin{aligned}
& \operatorname{Pr}\{\tau=k\}=\left\{\begin{array}{l}
y, k=1, \\
\frac{A}{(k+x)^{\beta+2}}, \quad k=2,3 \ldots
\end{array}\right. \text { - for an improved modification of the model; }
\end{aligned}
$$

where $x$ and $y$ are constants to be determined, $A$ is the normalization constant, and $\beta$ is the parameter related to the self-similarity ratio (Hurst parameter) with rate $\mathrm{H}=1-\beta / 2$ (Dann, 2006)..

Besides, each source has a certain rate of packets generation $S(n)$, in general case (basic model) $S(n)=1$. In the improved «Input $\mathrm{M} / \mathrm{G} / \infty »$ model, rate of packet generation is defined as follows:

$$
\operatorname{Pr}\{S=k\}=\frac{\operatorname{Pr}\left\{Y_{t}=k\right\}-\sum_{m=2}^{k} p_{m} \operatorname{Pr}\left\{\sum_{n=1}^{m} S_{n}=k\right\}}{p_{1}},
$$

where is the telecommunications traffic represented as a random process, value of which in each time slot is the total of intensity of information generation by all sources in the system at the moment: $Y_{t}$

$$
Y_{t}=\sum_{k=-\infty}^{t} \sum_{i=1}^{A_{t}} S_{k, i}(t-k) I\left(\tau_{k, i}>t-k\right)
$$

Coefficient $p_{k}$ is determined by the relation:

$$
\begin{gathered}
p_{0}=\operatorname{Pr}\left\{Y_{t}=0\right\}, \\
\operatorname{Pr}\left\{Y_{t}=1\right\}=p_{1} \operatorname{Pr}\{S=1\},
\end{gathered}
$$

etc. A detailed derivation is given in works (Privalov and Blagov, 2011).

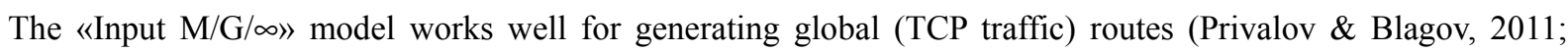
Blagov, 2011). An advanced «Input $\mathrm{M} / \mathrm{G} / \infty »$ model has a particular advantage in modeling traffic with statistical characteristics of small levels, like full-scale traffic characteristics (Privalov \& Blagov, 2011).

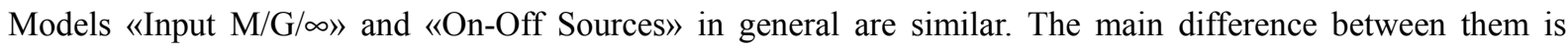
primarily that the first of them at any given time in the system may be an infinite number of sources, and the number of sources of the second is strictly limited.

The model «On-Off Sources» has $M$ independent sources. Each of them has two states: active state (ON), in which the source generates data, and the silent state (OFF), in which the source generates nothing. These periods follow each other alternately. In each ON period, the source generates information with certain (different from the other periods) intensity (rate) that is equal to a random value $S_{i}$ :

$$
\operatorname{Pr}\{S=k\}=\frac{\operatorname{Pr}\left\{Y_{t}=k\right\}-\sum_{m=2}^{M} p_{m} \operatorname{Pr}\left\{\sum_{n=1}^{m} S_{n}=k\right\}}{p_{1}} .
$$

The probability of detecting an individual source in the active state and in a silent state is:

$$
P_{O N}=\frac{E[\tau]}{E[\tau]+E[v]}, \quad P_{O F F}=\frac{E[v]}{E[\tau]+E[v]},
$$

where $\tau$ is the duration of $\mathrm{ON}$ periods of sources, is a realization of a random process that has a heavy tail distribution (1) in the base model, (2) - in its improved version.

$v$ is the duration of OFF periods with Poisson distribution: $\operatorname{Pr}\{v=k\}=e^{-\lambda} \frac{\lambda^{k}}{k !}, k \in Z_{+}$.

As in the model «Input $\mathrm{M} / \mathrm{G} / \infty)$ each source has the rate of packet generation that is defined for each ON period. The «On-Off Sources» model works well for generating local networks (Ethernet route) (Privalov and Blagov, 
2011). In an improved modification of the «On-Off Sources» model, the OFF period has a Poisson distribution of time:

$$
P_{O F F}=\frac{M v}{M \tau+M v}=\frac{\lambda}{M \tau+\lambda}, \lambda=\frac{P_{O F F}}{P_{O N}} M \tau .
$$

Experimenting with this type of distribution, it can be argued that results of modeling statistical characteristics, such as sample average, sample variance, one-dimensional distribution and autocorrelation function are better than in case of geometric distribution of the silence period, which is characteristic for the «On-Off Sources» base model (Privalov and Blagov, 2011).

\section{Experiments and Results}

\subsection{Environment for Modeling}

Let us choose the most suitable software package for the modeling jitter. Currently, there are many simulation environments. The most popular are: AnyLogic, Omnet ++, NS-2, GPSS (Karpov, 2005; Varga, 1999; Kudryavcev, 2003). Each of them has certain features, pros and cons. Feature of AnyLogic software package is the fact that it has a graphical user interface, which certainly is a big plus, makes it possible to use Java for modeling (Karpov, 2005). The wide range of tools and libraries allows system users to create models in many areas of knowledge, including the field of telecommunications and information systems.

The AnyLogic environment makes it possible to support approaches with both discrete and continuous time simulation.

For transferring into a continuous-time environment, we will use an approach of the AnyLogic environment called «system dynamics».

Let the service parameter in the system be: $s_{p}=\frac{v}{\rho}$, where $v$ is assumed to be the amount of information in a single packet of the network traffic multiplied by the sample average of the tested trace traffic during a certain

time slot of the trace. Here $\rho$ is the system load factor $(0<\rho<1)$.

We shall use global telecommunications networks traffic traces (traces of TCP traffic) as tested material. Global TCP traces: dec-pkt-1.tcp, dec-pkt-2.tcp, dec-pkt-3.tcp, dec-pkt-4.tcp, LBL-PKT-4.TCP (Internet Traffic Archive) - presented by traces of hour-long recording traffic between Digital Equipment Corporation (DEC - American Computer Company) and the rest of the Internet (the first two) and trace of an hour-long record of traffic between Lawrence Berkeley Laboratory (National Lawrence Laboratory in Berkeley), and the rest of the Internet (the last). Let us denote them as: tr.1, tr.2, ..., tr.6, respectively. Traces of local Ethernet networks are presented by traces of hour-long traffic records from the collection of the Bellcore corporation (the company producing means of communication, and performing research in this area): BC-pAug.TL, BC-pOct.TL (Internet Traffic Archive), namely, traffic traces in Bellcore LAN reference line. Let us denote them as: eth.1 and eth.2. In addition to the above well-known traces, let's take for studying yet another Ethernet trace with hour-long record in the LAN of Metromax, and denote it as «eth.3».

\subsection{Building Jitter Distribution}

As a result of creating in the AnyLogic medium a system with a single labeled (experimental oscillation frequency - 1 packet per $10 \mathrm{~ms}$ ) and a background threads, we will obtain values of the jitter. For comparing jitters in natural and simulated traffics, let us build its normalized one-dimensional distribution. For traffic that

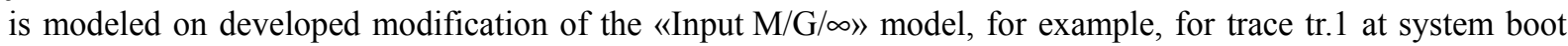
rate $\rho=0.8$ the following results are valid - see Figure 3. Implementations of jitter for natural continuous traffic and traffic generated by the base «Input M/G/ $/ \infty$ » model are shown in Figure 4. The resulting implementations of the jitter can demonstrate adequacy of the developed modification of the «Input $\mathrm{M} / \mathrm{G} / \infty$ » model for statistical characteristics of high levels, study of such characteristics as jitter requires, however, a more rigorous statistical assessment. 


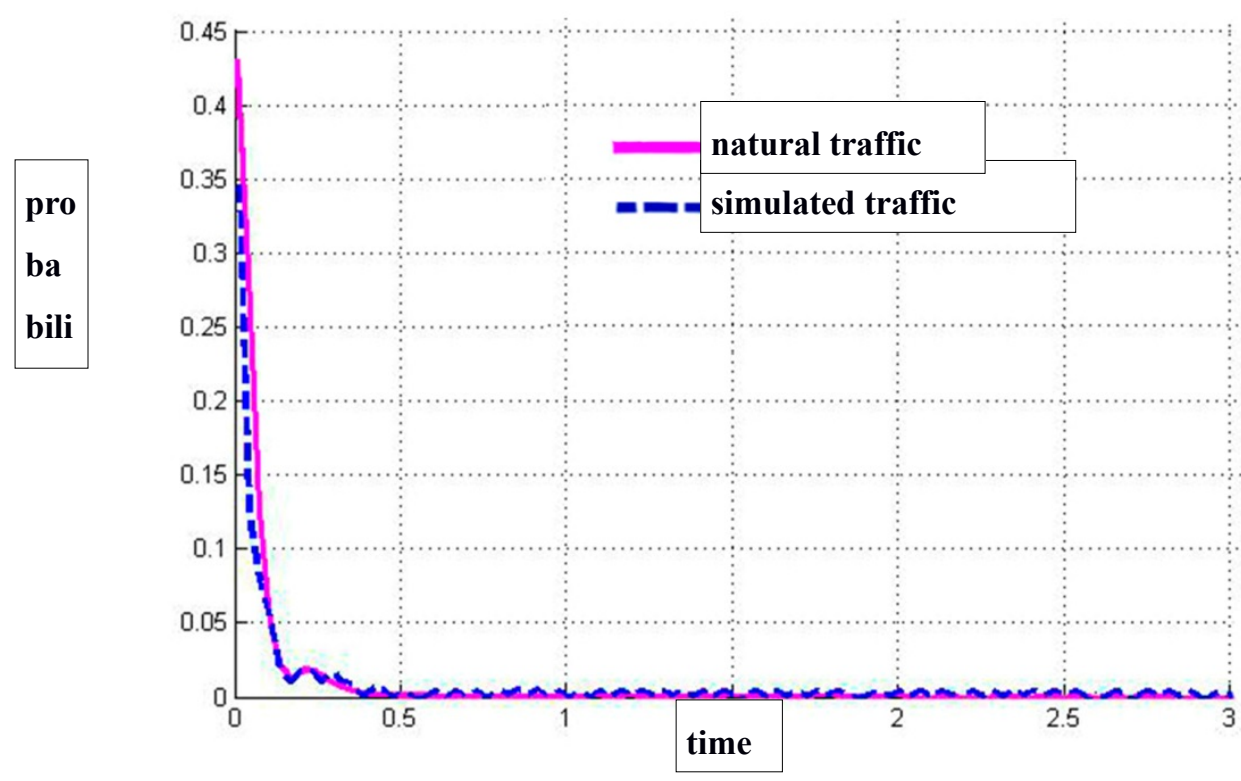

Figure 3. Distribution of jitter in natural traffic and the traffic modeled according to the developed modification of the «Input $\mathrm{M} / \mathrm{G} / \infty »$ model trace tr.1

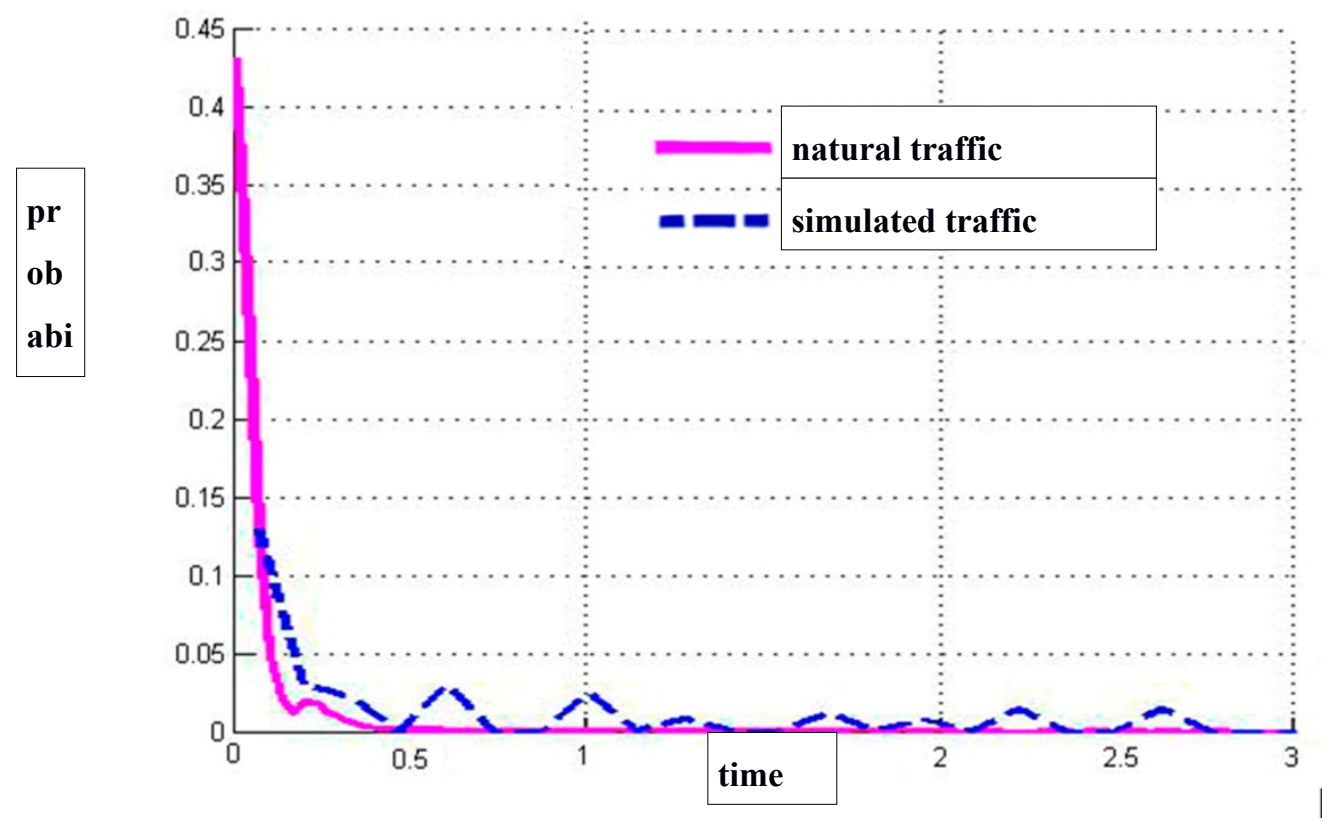

Figure 4. Distribution of jitter in natural traffic and the traffic modeled according to the basic «Input M/G/o»» model trace tr. 1

As for modeling jitter in LANs (eth1 and eth2), let us use model «On-Off Sources» in the experiment. Inadvisability of using the «Input $\mathrm{M} / \mathrm{G} / \infty$ » model in working with a LAN is proved in (Privalov and Blagov, 2011).

In course of system operation with a single labeled and background flow, generating in the basic and advanced modifications of the «On-off Sources» model, we have the following results (see Figures: 5, 6), for example, in working with trace eth.1. System load in this experiment we will also choose as $\rho=0.8$. According to the graph (see Figures 5 and 6), we can visually compare obtained distributions of jitter in implementation of an improved modification of the «On-off Sources» model and full-scale traffic routes LANs, as well as implementation of jitter traffic generated by the base model. 


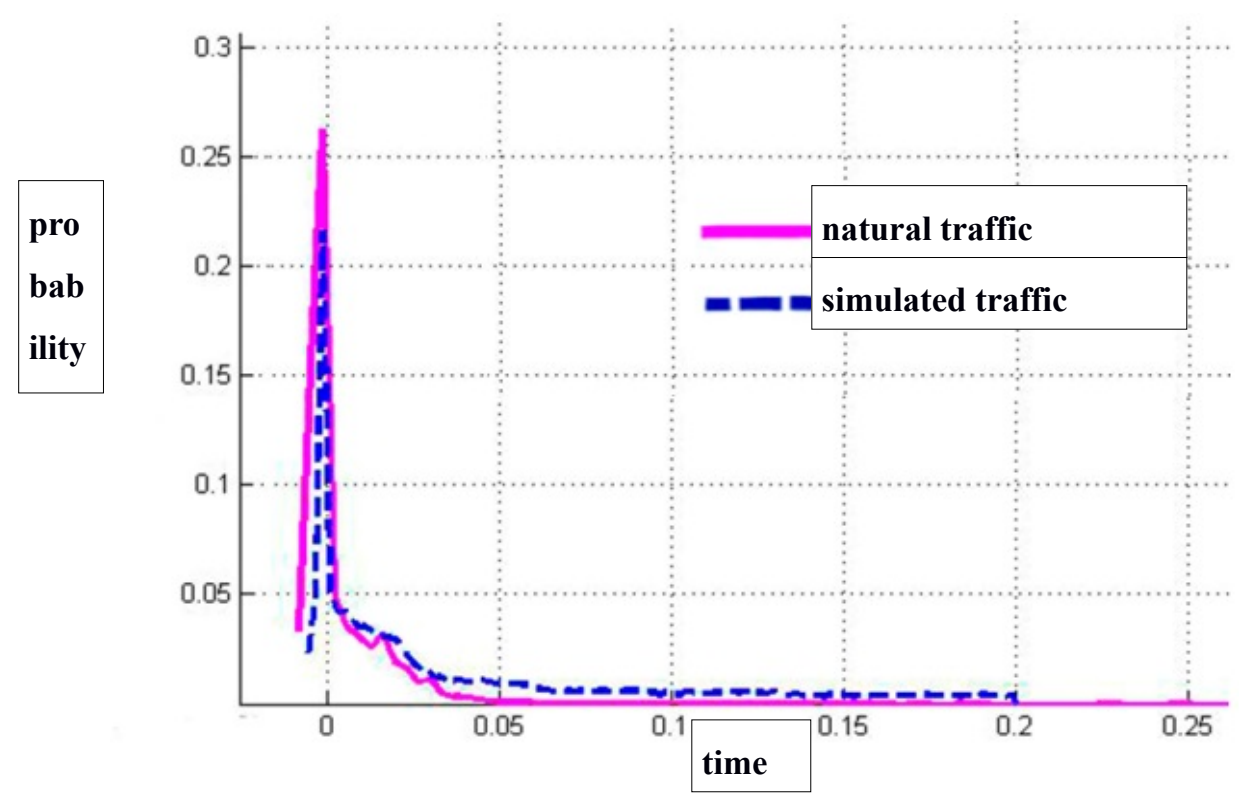

Figure 5. The distribution of jitter in natural traffic and traffic of trace eth.1 modeled on the developed modification of the «On-Off Sources» model

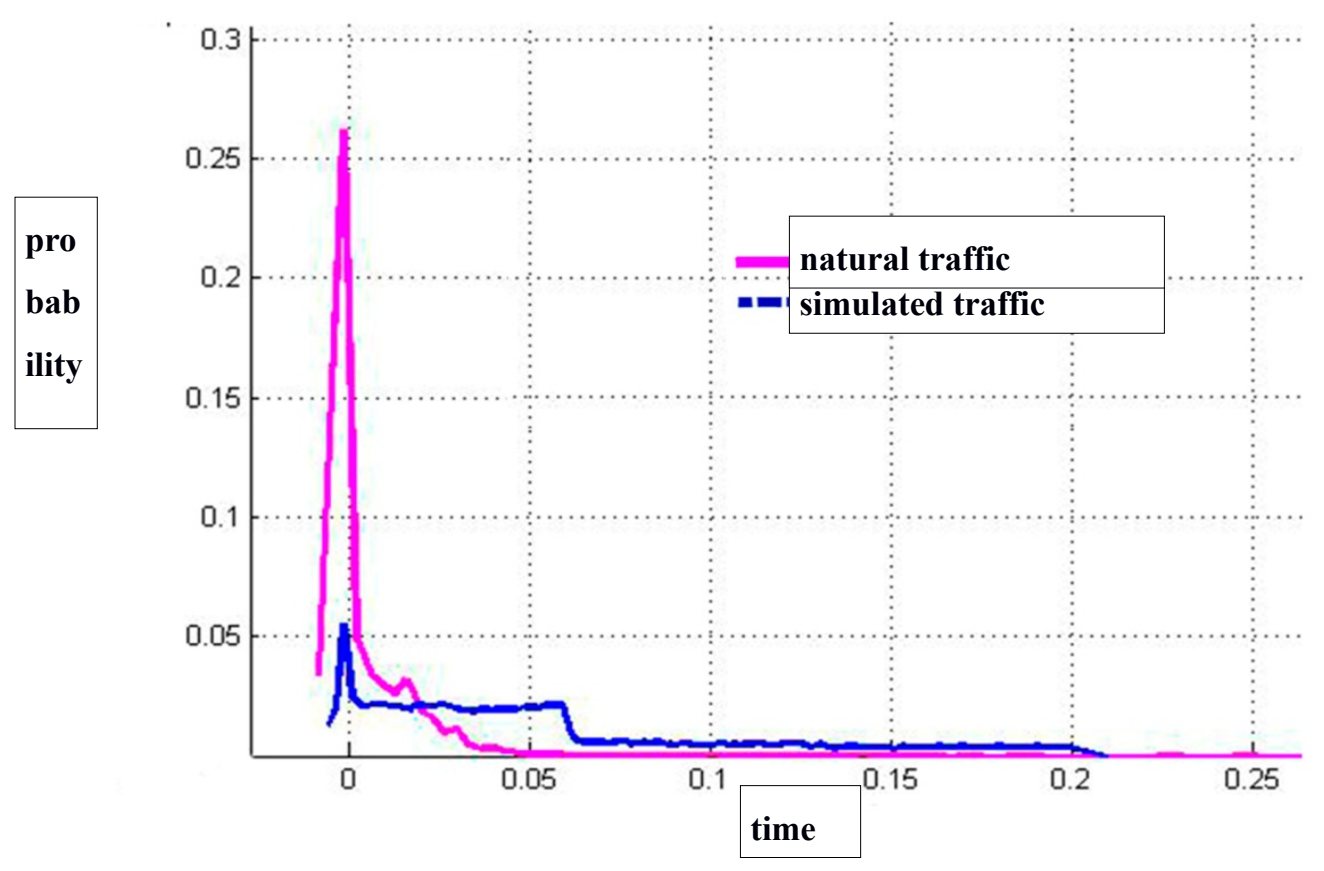

Figure 6. Distribution of jitter in natural traffic and traffic of the eth.1 trace modeled on basic «On-Off Sources» model

\subsection{Testing the Hypothesis}

Carry out checks that both samples (jitter distribution in natural and simulated traffic) obey the same distribution.

Using for this purpose the Kolmogorov-Smirnov test (used to determine whether the two independent samples $\left\{x_{1}^{(1)}, x_{2}^{(1)}, x_{3}^{(1)}, \ldots, x_{n}^{(1)}\right\}$ and $\left\{x_{1}^{(2)}, x_{2}^{(2)}, x_{3}^{(2)}, \ldots, x_{m}^{(2)}\right\}$ obey the same distribution of the random variable) (Kendall and Stuart, 1973). Statistics of this criterion is defined as: 


$$
D_{n, m}=\sup _{x}\left|F_{n}^{(1)}(x)-F_{m}^{(2)}(x)\right|,
$$

Where $F_{n}^{(1)}(x)=\sum_{i=1}^{n}\left(x_{i}^{(1)} \leq x\right)$ is the empirical function of distribution built on the first sample (in our case, on the one-dimensional distribution of natural traffic), and $F_{m}^{(2)}(x)=\sum_{i=1}^{m}\left(x_{i}^{(2)} \leq x\right)$ is the empirical distribution function built on the second sample (in our case, on the one-dimensional distribution of the simulated traffic).

Let us denote $H_{0}$ as the hypothesis that the two tested samples obey the same distribution of the random value (Gmurman, 1972). Then for introduced statistics:

$$
\forall t>0: \lim _{n, m \rightarrow \infty} \operatorname{Pr}\left(\sqrt{\frac{n m}{n+m}} D_{n, m} \leq t \mid H_{0}\right)=K(t),
$$

Where is $K(t)$ the Kolmogorov function.

If statistics of $\sqrt{\frac{n m}{n+m}} D_{n, m}$ exceeds the quantile of Kolmogorov distribution $t_{1-\alpha}$, the zero hypothesis $H_{0}$ is rejected with an error probability. Otherwise, the hypothesis is accepted at the level of significance $\alpha$.

Let us calculate statistics $\sqrt{\frac{n m}{n+m}} D_{n, m}$ for our problem, and then compare it to quantiles of distribution $t_{1-\alpha}$, thereby accepting or rejecting the hypothesis $H_{0}$ that the two tested samples obey the same distribution at the level of significance $\alpha$ or with probability of error $\alpha$, respectively. Values for this statistics for various tested traces of global networks (let us denote them as tr1, tr2, tr3, tr4 and tr5) we will write into Table 1.

Table 1. Value of jitter distribution statistics in global traces according to the Kolmogorov-Smirnov test

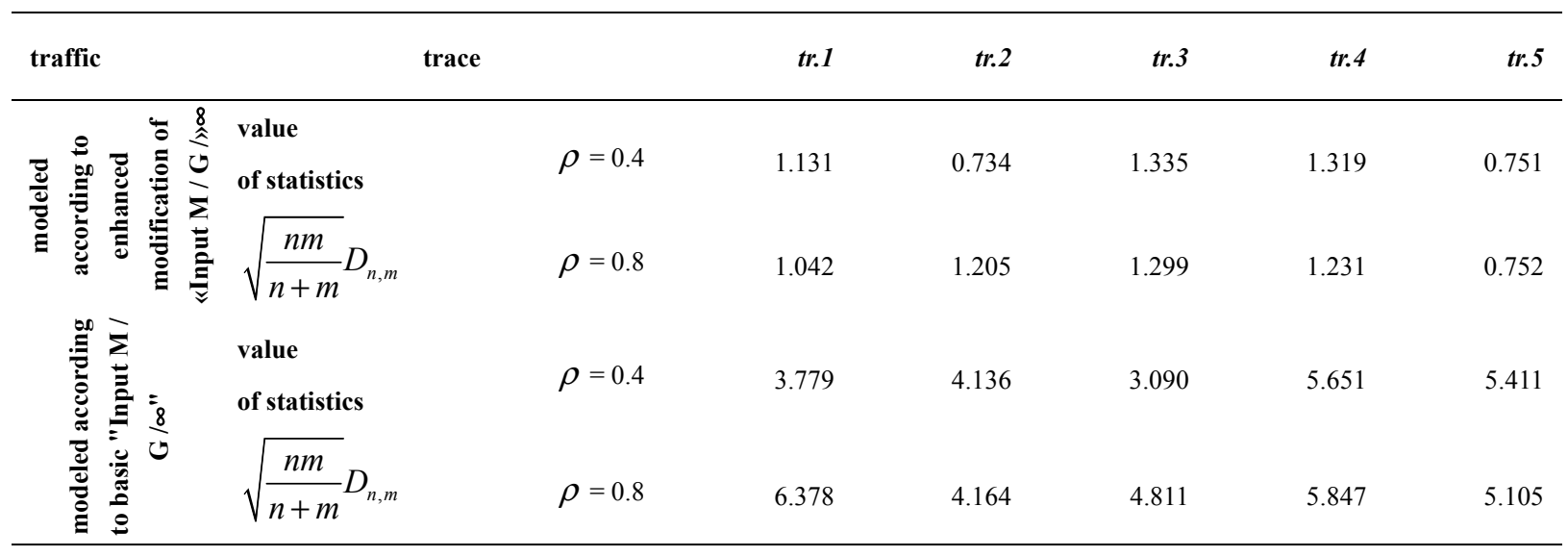

According to the results presented in Table 1, it can be concluded that the hypothesis $H_{0}$ that the two tested samples obey the same distribution; is taken at the significance level 0.05 by the Kolmogorov-Smirnov criterion (Bolshev and Smirnov, 1983) in the case of with jitter distribution in natural traffic and in the traffic modeled according to the developed modification of the «Input $\mathrm{M} / \mathrm{G} / \infty$ » model with the approximation of one-dimensional distribution and autocorrelation function. For the case of the traffic received from the basic «Input $\mathrm{M} / \mathrm{G} / \infty$ » model without approximation, the hypothesis $H_{0}$ is rejected (obtained statistic values are greater than distribution quantile). At the same time, both accuracy of defining sample average and jitter sample dispersion are on the average 3-4 times higher than for the developed modification of the model.

Same as in case of global traces, let us inspect implementations of jitter distribution in natural and simulated traffic by the Kolmogorov-Smirnov test (let us write statistics into Table 2). 
Table 2. Value of jitter distribution statistics in local traces according to the Kolmogorov-Smirnov test

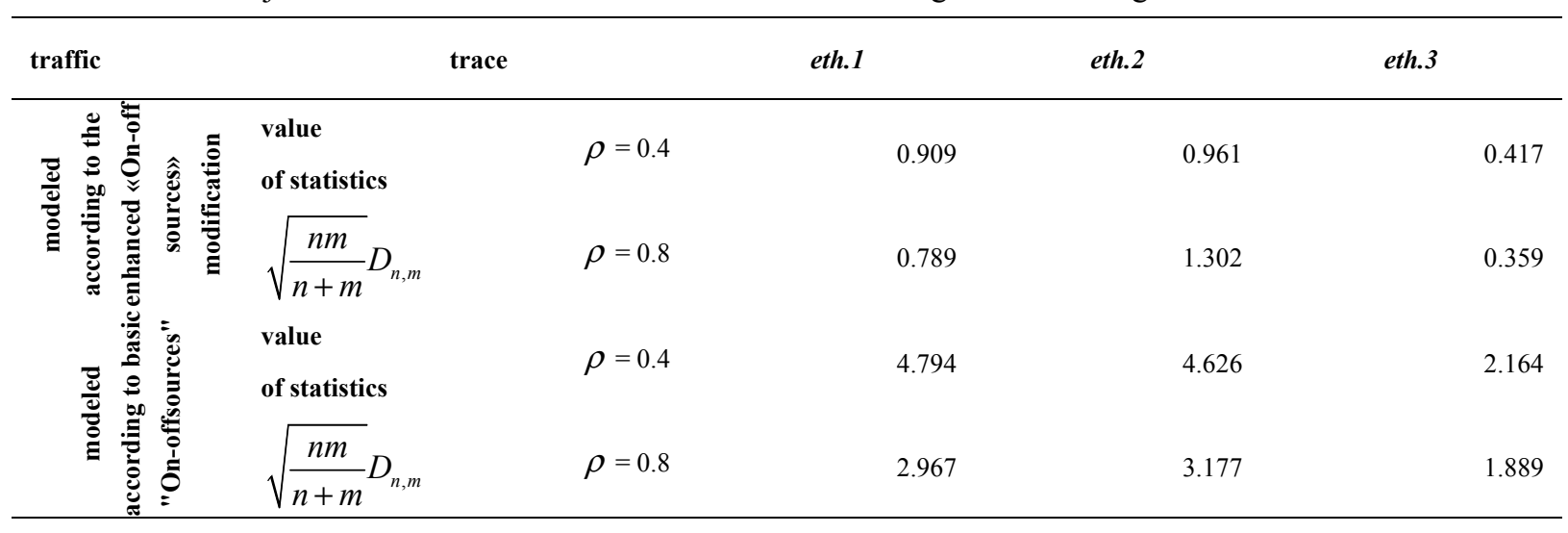

Same as is the case with the «Input M/G/» model, jitter distribution in traffic modeled according to the improved «On-off Sources» modification is identical to jitter distribution in natural traffic by selected criteria. The hypothesis that the two test samples obey the same distribution is taken at the level of significance 0.05 , the criterion of the Kolmogorov-Smirnov test. In the case of traffic modeled according to the base model, the hypothesis is rejected.

According to obtained jitter implementations adequacy of the developed modification of the «On-off Sources» model can be judged by the statistical characteristic of high level - jitter.

\section{Discussion, Conclusions and Perspectives}

Performed experiments for assessing delay variation - jitter in natural and simulated traffic demonstrates the benefits of certain models of network traffic before others. As a result of comparing improved modifications of «Input $\mathrm{M} / \mathrm{G} / \infty$ » and «On-off Sources» models to the basic models it can be said that the former have advantage over the latter when checking for jitter in situations similar to real, obtained in an environment with continuous time and wide range of the AnyLogic tools. It means that the modeling according to developed modifications of models makes it possible to analyze Quality of Service in a telecommunications network by the characteristic of delay variation - jitter. The AnyLogic simulation environment provides, in turn, a convenient tool for building simulation models with continuous time. With that, the «Input $\mathrm{M} / \mathrm{G} / \infty$ » model is more applicable for simulation of global networks traces, and «On-off Sources» - for local.

The paper showed the opportunity to simulate network telecommunications traffic by improved modifications if tested «Input M/G/œ» and «On-off Sources» models with preserving QoS parameters, namely, delay variation. Distribution of jitters in natural and model traffic obey the same distribution by the Kolmogorov-Smirnov test.

As for modeling the jitter itself, the approach proposed in this paper makes it possible to both determine the variation of network delay, and to explain its physical nature. However, this approach only allows obtaining some assessment of the quality of service in a network without making concrete recommendations for improving it, which in turn requires use of additional methods and tools.

\section{Acknowledgements}

This work was supported by the Ministry of Education and Science of the Russian Federation in the framework of the implementation of the Program of increasing the competitiveness of SSAU among the world's leading scientific and educational centers for 2013-2020 years.

\section{References}

Blagov, A. V. (2011). Building imitation models of self-similar telecommunications traffic. Bulletin of the Samara State Aerospace University named after academician S.P. Korolyov, 2(26), 201-210.

Bolshev, L. N., \& Smirnov, N. V. (1983). Tables of Mathematical Statistics (pp. 416). Moscow: Nauka.

Dunn, J. (2006). Jitter. Theory. Part 1. iXBT.com. Retrieved July 18, 2014, from http://www.ixbt.com/proaudio/jitter-theory-part1.shtml

Erramilli, A., Narayan, O., \& Willinger W. (1996). Experimental queuing, analysis with long-range dependent pocket traffic IEEE. ACM Trans. Networking, 4(2), 209-223. http://dx.doi.org/10.1109/90.491008 
Gmurman, V. E. (1972). The theory of probability and mathematical statistics (pp. 480). Moscow: Vysshaya Shkola.

Gromov, Y. Y., Zemskoy, N. A., Ivanova, O. G., \& Lagutin, A. V. (2007). Fractal analysis and processes in computer networks. Tambov: Publishing House of Technical University.

Internet Traffic Archive. Retrieved September 21, 2011, from http:/ita.ee.lbl.gov/html/traces.html

Karpov, Y. G. (2005). Simulation modeling of systems. Introduction into modeling in AnyLogic 5 (pp. 403). St. Petersburg: BHV-Petersburg Publishing House.

Kendall, M., \& Stuart, A. (1973). Statistical conclusions and relations (pp. 323). Moscow: Nauka.

Kudryavtsev, E. M. (2003). GPSS World. Fundamentals of simulation modeling of various systems (pp. 320). Moscow: DMK Press.

Matrawy, A., Lambadaris, I., \& Huang, C. (2002). MPEG4 traffic modeling using the transform expand sample methodology (pp. 248-256). In the Proceedings of $4^{\text {th }}$ IEEE. http://dx.doi.org/10.1109/IWNA.2001.980870

Melamed, B., Donatiello, L., \& Nelson, R. (2003). An overview of TES process and modeling methodology: Models and techniques for Performance Evaluation of Computer and Communications Systems (pp. 359-393). New York: Springer Verlag. http://dx.doi.org/10.1007/BFb0013860

Mondragon, R. J., Arrowsmith, D. K., \& Pitts, J. M. (2001). Chaotic map for traffic modeling and queuing analysis. $\quad$ Netherlands: $\quad$ PerformanceEvaluation, $223-240$. http://dx.doi.org/10.1016/S0166-5316(00)00047-X

Park, K., Willinger, W., \& Wiley, Ed. (2000). Self-similar Network Traffic and Performance Evaluation (pp. 556). New York: JohnWiley\&Sons, Inc.

Petrov, V. V. (2004). Teletraffic structure and algorithm of QoS provision under the influence of the self-similarity effect (pp. 197). Moscow: Publishing house of the Energy Institute.

Privalov, A. Y. (2000). Two-point jitter in a network of periodic flows under the maximum load. Problems of information transfer, 36, 96-111. Retrieved from http://mi.mathnet.ru/eng/ppi479

Privalov, A. Y. (2007). Jitter smoothing constant bit rate flow after one or several nodes. Problems of information transfer, 43, 85-111. Retrieved from http://mi.mathnet.ru/eng/ppi14

Privalov, A. Y., \& Blagov, A. V. (2011). About using some models of self-similar network traffic in imitation simulation. Mathematical modeling, 23(7), 114-128. Retrieved from http://mi.mathnet.ru/eng/mm3135

Ramakrishnan, P. (1997). Self-similar traffic model: Technical Report CSHCN T.R.99-5 (ISR T.R. 99-12) (pp. 26). Maryland: Center for Satellite and Hybrid Communication, University of Maryland.

Shelukhin, O. I., Tenyakshev, A. M., \& Osin, A. V. (2003). Fractal processes in telecommunications (pp. 480). Moscow: Radiotekhnika.

Varga, A. (1999). Using the Omnet++ discrete event simulation system in education (pp. 11). In IEEE Transaction on Education.University of New Mexico. http://dx.doi.org/10.1109/13.804564

Vegesna, S. (2002). IP Quality of Service (pp. 368). PublishingHouse «Williams».

\section{Copyrights}

Copyright for this article is retained by the author(s), with first publication rights granted to the journal.

This is an open-access article distributed under the terms and conditions of the Creative Commons Attribution license (http://creativecommons.org/licenses/by/3.0/). 